\title{
Symptomatic Tongue Atrophy Due to Atypical Polymyositis in a Pembroke Welsh Corgi
}

\author{
Daisuke ITO ${ }^{1)}$, Midori OKADA ${ }^{2,5)}$, Nick D. JEFFERY ${ }^{3)}$, Masato KITAGAWA ${ }^{1) *}$, Kazuyuki UCHIDA ${ }^{4)}$ and \\ Toshihiro WATARI ${ }^{1)}$ \\ ${ }^{1)}$ Nihon University, School of Veterinary Medicine and ${ }^{2)}$ Nihon University, School of Veterinary Research Center, 1866 Kameino, \\ Fujisawa, Kanagawa 252-8510, Japan, ${ }^{3)}$ Department of Veterinary Medicine, University of Cambridge, Cambridge CB3 OES, United \\ Kingdom, ${ }^{4}$ Department of Veterinary Pathology, Graduate School of Agricultural and Life Sciences, The University of Tokyo, 1-1-1 \\ Yayoi, Bunkyoku, Tokyo 113-8657 and ${ }^{5)}$ Pet Clinic ANIHOS, 1-14-11 Minamitokiwadai, Itabashi, Tokyo 174-0072, Japan
}

(Received 14 November 2008/Accepted 4 March 2009)

ABSTRACT. A three-year-old, 7.3-kg, female Pembroke Welsh Corgi exhibited symptomatic tongue atrophy, crinkling of the tip of the tongue, dysphagia and excessive salivation. Neurological examination suggested multiple cranial neuropathy, but polymyositis was diagnosed by magnetic resonance imaging and muscle biopsy. The dog did not respond to prednisolone treatment and died from aspiration pneumonia 22 months after the first presentation. Post-mortem histopathological examination of the tongue revealed marked myofiber loss and fibrosis with multifocal infiltration of mixed mononuclear cells. Similar findings were also observed in the masticatory muscles and quadriceps without abnormality of peripheral nerves or evidence of infection. Symptomatic tongue atrophy occurring in the course of polymyositis has not been reported previously in dogs.

KEY WORDS: canine, dog, myopathy, myositis.

Idiopathic polymyositis is one type of noninfectious generalized inflammatory myopathy and is diagnosed by a combination of abnormalities including i) clinical signs of muscle weakness, ii) abnormally high serum muscle enzyme including creatine kinase (CK) and aspartate aminotransferase (AST), iii) abnormal electromyography (EMG) findings, iv) negative serologic test for infectious disease and v) histological evidence of myopathy and infiltration of lymphocytes within affected muscles [7, 13]. Typically, it has an immune-mediated aetiology $[8,11]$.

In dogs, the incidence of tongue involvement in cases of polymyositis is unknown although atypical polymyositis primarily affecting extraocular muscles or the right atrium have previously been reported $[5,16]$. Furthermore, in humans, tongue myositis is rarely a component of inflammatory muscle disease [6]. This case report is the first description of a dog (Pembroke Welsh Corgi) in which there were clinical signs of severe tongue atrophy resulting from polymyositis focused specifically on the tongue and masticatory muscles.

A three-year-old, 7.3-kg, female Pembroke Welsh Corgi was presented to the Animal Medical Center (ANMEC) at Nihon University, with the complaint of atrophy of masticatory muscles and the tongue, dysphagia and excessive salivation. The clinical signs of excessive salivation and abnormal movement of the tongue were first apparent two years prior to presentation, when the dog was one year old. At that time, the owner also noted that the dog occasionally had difficulty swallowing. Three months prior to referral,

\footnotetext{
* Correspondence to: Dr. Kitagawa, M., School of Veterinary Medicine, Nihon University, 1866 Kameino, Fujisawa, Kanagawa 252-8510, Japan.

e-mail : kitagawa@brs.nihon-u.ac.jp
}

atrophy and crinkling of the tongue, dysphagia, weight loss of $2 \mathrm{~kg}$ and atrophy of the masticatory muscles were noted by the owner and referring veterinarian. Initially, the referring veterinarian suspected trigeminal, facial, hypoglossal, glossopharyngeal and vagal neuropathies, perhaps including masticatory muscle myositis (MMM), and treated the dog with prednisolone $\left(1 \mathrm{mg} / \mathrm{kg} / \mathrm{day}\right.$, Predonin ${ }^{\circledR}$, Shionogi \& Co., Ltd., Tokyo, Japan) for 2 weeks, which was then decreased gradually over the next 2 weeks (half dose for the 1 st week, quarter dose for the next seven days and then terminated because of lack response to the treatment).

At presentation at ANMEC, physical examination revealed atrophy of the tongue, crinkling of the tip of the tongue and mild bilateral atrophy of the masticatory muscles, but other muscles, including those of the limbs, appeared unaffected (Fig. 1). The dog's rectal temperature was slightly increased $\left(39.8^{\circ} \mathrm{C}\right)$, and muscle pain was not detected on palpation. Neurological examination revealed dysphagia, a weak palpebral reflex and menace reaction. The results of routine haematological examination were unremarkable (there was no eosinophilia), but the levels of CK (1,700 U/L; reference range 10-200 U/L) and AST (221 $\mathrm{U} / \mathrm{L}$; reference range $0-50 \mathrm{U} / \mathrm{L})$ were elevated. The dog's antinuclear antibody (ANA) and serum distemper virus antibody titers were negative.

Magnetic resonance imaging (MRI) was performed under general anesthesia using a 1.5-T MRI system (Toshiba, EXCELART Vantage, Tokyo, Japan) to investigate the possibility of intracranial disease affecting the cranial nerves. No abnormalities were found in the nervous system, but on T1-weighted (T1W), T2-weighted (T2W) and FLAIR images revealed hyperintense areas in the ventral part of the temporalis muscle bilaterally, which was thought to be 


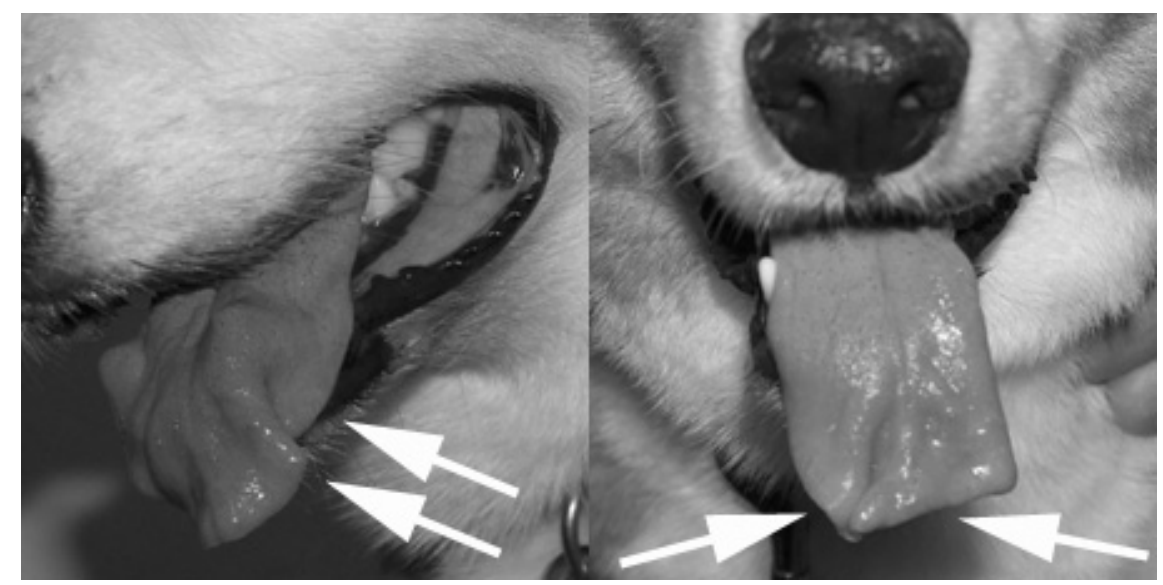

Fig. 1. Appearance of the case. The tongue is thin, causing crinkling and turning at the tip (arrows).

caused by fat replacement (Fig. 2). In the area adjacent to these abnormal findings, there was hyperintensity on $\mathrm{T} 2 \mathrm{~W}$ and FLAIR images thought to indicate inflammation and oedema. These areas had normal intensity on $\mathrm{T} 1 \mathrm{~W}$ images but were enhanced following intravenous administration of the nonionic contrast agent gadoteridol (ProHance, BraccoEisai Co., Ltd., Tokyo, Japan) at a dose of $0.3 \mathrm{ml} / \mathrm{kg} / \mathrm{I}$.V. The tongue could not be evaluated with MRI because of poor structural definition. Cerebral spinal fluid (CSF) collected from the cisterna magna revealed no abnormality of cell or protein content, and the distemper virus antibody titer was zero. Electromyography (EMG) of all examined muscles, including masticatory muscles, tongue and the quadriceps, revealed no abnormalities.

Serum tested by the Comparative Neuromuscular Laboratory, University of California, San Diego showed insignificant concentrations $(<1: 100)$ of type $2 \mathrm{M}$ autoantibodies (versus masticatory muscle) and antibodies against the acetylcholine (ACh) receptor (i.e., negative test for immunemediated myasthenia gravis).

Biopsies of the temporalis, masseter and quadriceps muscles were submitted to the Comparative Neuromuscular Laboratory for histopathological investigation. The temporalis muscle showed marked myofiber loss, fatty infiltration and fibrosis in some areas of the muscle, with other areas showing myofiber atrophy. Multifocal accumulations of mixed mononuclear cell infiltrations were present and had an endomysial and perivascular distribution. The masseter muscle showed similar findings to the temporalis muscle but without fibrosis and fiber loss. No specific abnormalities were identified within the biopsy sample of quadriceps muscle.

In view of the tongue and masticatory muscle atrophy, clinical and histopathological findings plus the negative type $2 \mathrm{M}$ antibody test, a diagnosis of polymyositis was considered most probable, and prednisolone was prescribed at an immune-modifying dose $(2 \mathrm{mg} / \mathrm{kg} /$ day/S.C. $)$. After three weeks of treatment, the dysphagia and tongue atrophy had not improved. Further treatment with prednisolone $(1 \mathrm{mg} /$ $\mathrm{kg} /$ day/S.C.) for six weeks resulted in no alteration of the dog's condition, and prednisolone treatment was terminated. Progression of masticatory muscle atrophy and dysphagia were observed a month after withdrawal of prednisolone. In addition, at approximately five months after the first presentation at ANMEC, the dog showed dysarthria, absence of palpebral reflex and menace reaction and aspiration pneumonia with bacteria. Therefore, amoxicillin (20 mg /kg, bid, Pasetocin ${ }^{\circledR}$, Kyowa Hakko Kogyo Co., Ltd., Tokyo, Japan) and an ophthalmic ointment containing erythromycin and colistin (optimal dose, Ecolicin ${ }^{\circledR}$ ophthalmic ointment, Santen Pharmaceutical Co., Ltd., Osaka, Japan) were used according to the clinical signs. At approximately 12 months after first presentation, the dog showed regurgitation and dysphonia with progression of the other clinical signs. At 18 months after first presentation, the dog showed severe dysphagia due to laryngopharyngeal paralysis and paresis of tongue movement, and had lost an additional $2 \mathrm{~kg}$ body weight (because of difficulty eating), frequent regurgitation and slight pelvic limb gait abnormality. The dog finally died 22 months after first presentation because of aspiration pneumonia (diagnosed by autopsy and histopathology).

At post-mortem examination, the temporalis and masseter muscles appeared generally similar in histological appearance to the biopsies, although the changes were more severe (Fig. 3). Severe myositis was also identified in the tongue (Fig. 3). In addition, the quadriceps muscle, which previously had been unaffected, exhibited patchy regions of moderate myofiber loss and fibrosis with infiltration of mixed mononuclear cells (Fig. 3). There was no evidence of Protozoal infection including Toxoplasma, Neospora or Leishmania. Peripheral nerve tissue appeared normal.

We initially suspected peripheral neuropathy affecting multiple cranial nerves because of the dysphagia, abnormal movement of the tongue, weakness of the palpebral reflex and menace response without clinical signs of generalized 

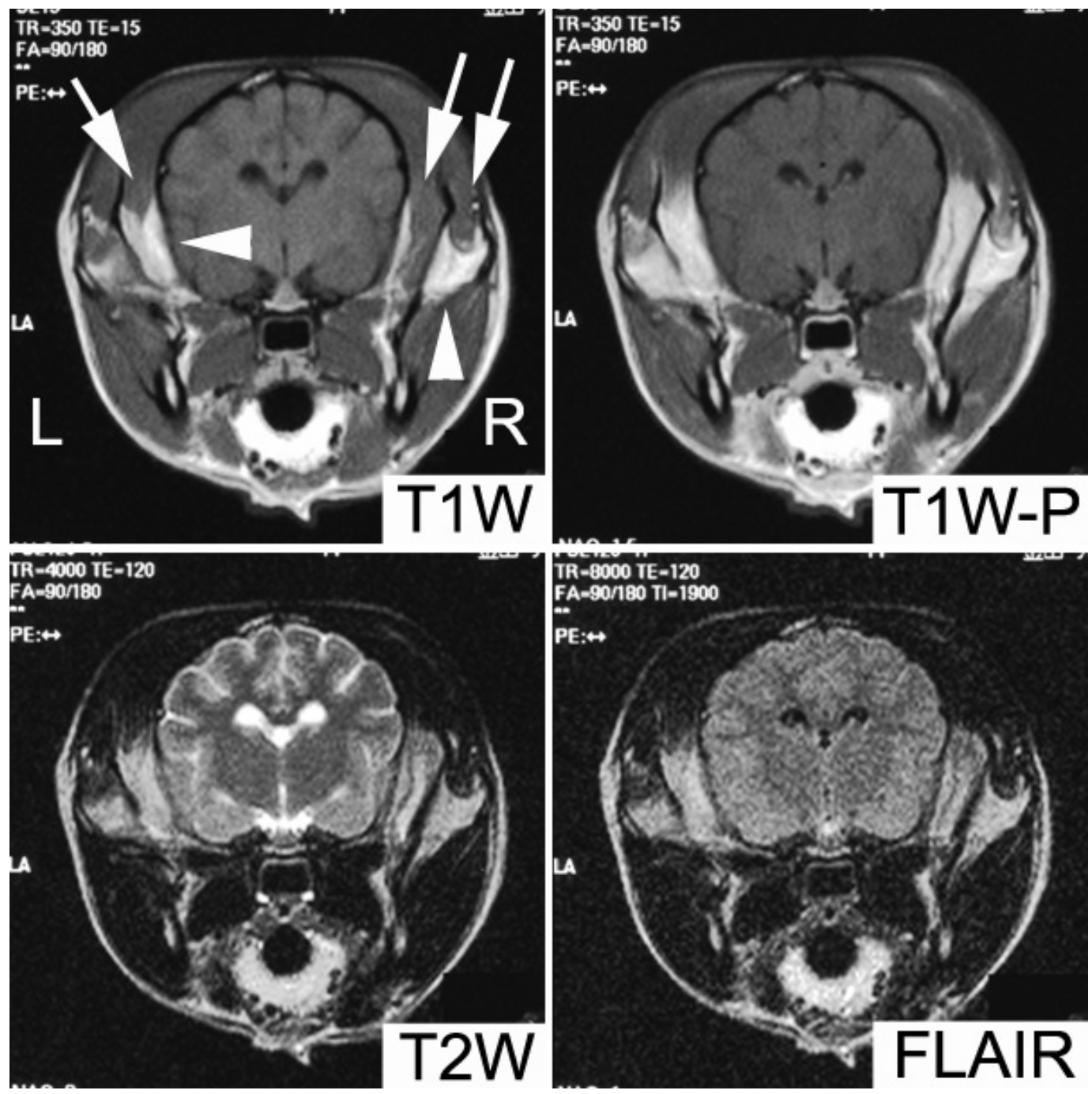

Fig. 2. Transverse magnetic resonance images (MRI) at the level of the thalamus. On T1-weighted (T1W), T2weighted (T2W) and FLAIR images, there are hyperintense areas in the ventral part of the temporalis muscle (arrow heads). In the area adjacent to these abnormal findings, there is hyperintensity on T2W and FLAIR images. These areas had normal intensity on T1W images (arrows) and were enhanced following intravenous administration of the nonionic contrast agent gadoteridol (ProHance). T1W-P: T1-weighted-ProHance.

muscle disease. However, polymyositis was diagnosed because of the following findings: i) negative results for autoantibodies of type $2 \mathrm{M}$ muscle fibers, and ii) histopathological evidence of infiltration of mononuclear cells into skeletal muscles without evidence of infectious disease or abnormalities of the cranial nerves. The lack of inflammation in the biopsied quadriceps muscles at an early stage is puzzling, but two reasons can be suggested. One is that the biopsied samples were too small and did not include lesions. The other is that myositis developed in the quadriceps muscles during the two years after first occurrence of the disease.

A similar case of polymyositis affecting the tongue and causing macroglossia in a human patient has been reported in a single report [6]. Clinical signs of slight muscle atrophy in all 4 limbs, pain on muscle pressure and macroglossia were seen at an early stage and progressed to dysarthria, frequent tongue biting during mastication, dysphagia and noisy breathing. Although clinical signs appeared at differing stages throughout the course of disease in both the human patient and this dog, both exhibited slight muscle atrophy in limbs, abnormal tongue movement, dysphonia and dysphagia. However, in the human patient, oropharyngeal symptoms improved greatly within a few days of administration of high doses of prednisone $(0.7 \mathrm{mg} / \mathrm{kg} / \mathrm{day})$, which did not occur in the dog. Furthermore, there were differences in the effect of the condition on the tongue size and pain; the human patient showed macroglossia plus pain, but the dog showed tongue atrophy without pain, suggesting the possibility that the acute phase was not noticed in the dog. 

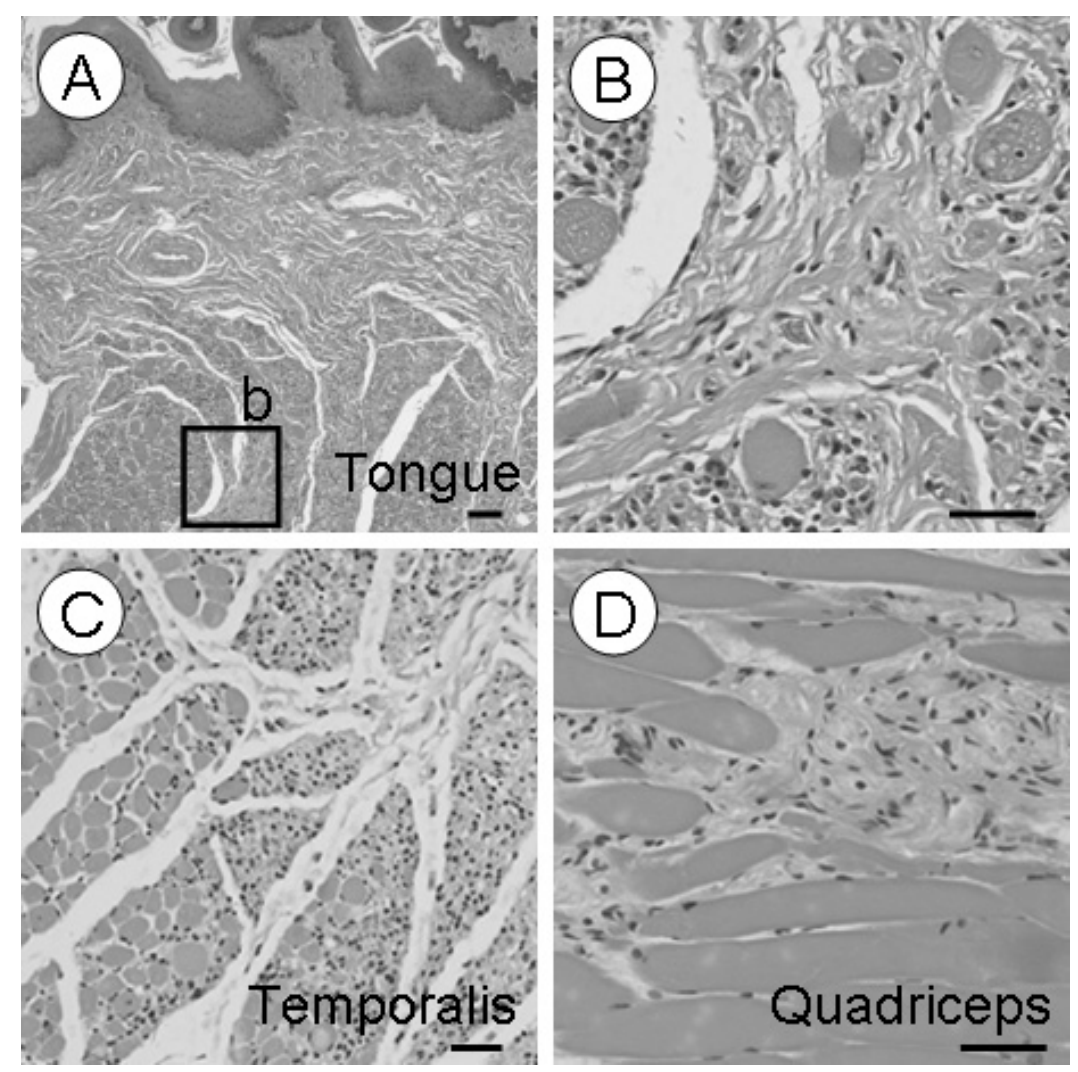

Fig. 3. Fresh-frozen sections from the tongue (A, B), temporalis (C) and quadriceps (D). A, B: There was severe myofiber atrophy and loss, perimysial and endomysial fibrosis and multifocal accumulations of mixed mononuclear cells infiltrations having an endomysial and perivascular distribution in the tongue (B: boxed area from A). C: Mild to severe myofiber atrophy and loss, fibrosis and mixed mononuclear cells infiltrations within the temporalis muscle. D: Slight endomysial fibrosis with infiltrations of mixed mononuclear cells within the quadriceps muscle. Hematoxylin-eosin (HE) stain. The bars represent $20 \mu \mathrm{m}$.

Although the human patient was treated effectively, treatment was ineffective in the present case, perhaps because of disease chronicity. There was a 2-year period between the onset of clinical signs and the first use of prednisolone. At the chronic stage of myositis, muscle becomes atrophied, infiltrated with fat, connective and fibrous tissue [1]. These changes are irreversible, and it is known that fatty infiltration is not responsive to corticosteroid therapy in either humans or dogs $[1,8,12]$. Therefore, Evans et al. suggested that early and appropriate treatment would be most likely to produce the optimum outcome [8].

The results of electromyography (EMG) were not abnormal in this dog, perhaps because of fibrosis and fatty infiltration, which has been reported to reduce EMG activity [8, $12,15]$. In addition, it is thought that the amount of abnormal activity reflects the degree of active inflammation in humans [2, 4], and it has been reported that corticosteroids reduce abnormal EMG findings in dermatomyositis and polymyositis [3]. Therefore, it is possible that the prior use of corticosteroids may also have been responsible for the nor- mal EMG findings in this dog.

In humans, MRI findings in inflammatory myopathies, including polymyositis, are well established $[1,9,10,12]$. It has been suggested that muscle oedema and fatty infiltration are signs of chronic inflammation of muscle in humans [1, 12]. Similar to human studies, evidence of oedema and fatty infiltration within the temporalis muscle was observed symmetrically on MRI in the present case.

In this case, the findings of severe myositis were restricted to masticatory muscles and the tongue, although mild myositis was also seen in the quadriceps. This suggested that the localization of severe polymyositis involves an immune response directed against specific muscle fiber antigens in the masticatory muscles and tongue similar to that of previously reported cases of extraocular polymyositis [5], myocarditis with polymyositis [16] and MMM [8, 14].

In conclusion, this is the first report of a dog with symptomatic severe atrophy of the tongue because of concurrent polymyositis. 


\section{REFERENCES}

1. Adams, E. M., Chow, C. K., Premkumar, A. and Plotz, P. H. 1995. The idiopathic inflammatory myopathies: spectrum of MR imaging findings. Radiographics 15: 563-574.

2. Amato, A. A. and Barohn, R. J. 1997. Idiopathic inflammatory myopathies. Neurol. Clin. 15: 615-648.

3. Blijham, P. J., Hengstman, G. J., Hama-Amin, A. D., Van Engelen, B. G. and Zwarts, M. J. 2006. Needle electromyographic findings in 98 patients with myositis. Eur. Neurol. 55: 183-188.

4. Bohan, A., Peter, J. B., Bowman, R. L. and Pearson, C. M. 1977. Computer-assisted analysis of 153 patients with polymyositis and dermatomyositis. Medicine 56: 255-285.

5. Carpenter, J. L., Schmidt, G. M., Moore, F. M., Albert, D. M., Abrams, K. L. and Elner, V. M. 1989. Canine bilateral extraocular polymyositis. Vet. Pathol. 26: 510-512.

6. Chauvet, E., Sailler, L., Carreiro, M., Paoli, J.R., Arrue, P., Astudillo, L., Oksmann, F., Delisle, M.B. and Arlet, P. 2002. Symptomatic macroglossia and tongue myositis in polymyositis: treatment with corticosteroids and intravenous immunoglobulin. Arthritis Rheum. 246: 2762-2764.

7. Engel, A. G. and Hohlfeld, R. 2004. The polymyositis and dermatomyositis syndromes. pp. 1321-1366. In: Myology, (Engel, A. G. and Franzini-Armstrong, C.) McGraw-Hill, New York.
8. Evans, J., Levesque, D. and Shelton, G. D. 2004. Canine inflammatory myopathies: a clinicopathologic review of 200 cases. J. Vet. Intern. Med. 18: 679-691.

9. Fleckenstein, J. L. and Reimers, C. D. 1996. Inflammatory myopathies: radiologic evaluation. Radiol. Clin. North Am. 34: 427-439, xii.

10. Garcia, J. 2000. MRI in inflammatory myopathies. Skeletal Radiol. 29: 425-438.

11. Kornegay, J. N., Gorgacz, E. J., Dawae, D. L., Bowen, J. M., White, N. A. and Debuysscher, E. V. 1980. Polymyositis in dogs. J. Am. Vet. Med. Assoc. 176: 431-438.

12. May, D. A., Disler, D. G., Jones, E. A., Balkissoon, A. A. and Manaster, B. J. 2000. Abnormal signal intensity in skeletal muscle at MR imaging: patterns, pearls, and pitfalls. Radiographics 20: 295-315.

13. Podell, M. 2002. Inflammatory myopathies. Vet. Clin. North Am. Small Anim. Pract. 32: 147-167.

14. Shelton, G. D., Cardinet, G. H. 3rd and Bandman, E. 1987. Canine masticatory muscle disorders: a study of 29 cases. Muscle Nerve. 10: 753-766.

15. Taylor, S. M. 2000. Selected disorders of muscle and the neuromuscular junction. Vet. Clin. North Am. Small Anim. Pract. 30: $59-75$.

16. Warman, S., Pearson, G., Barrett, E. and Shelton, G. D. 2008. Dilatation of the right atrium in a dog with polymyositis and myocarditis. J. Small Anim. Pract. 49: 302-305. 